\title{
Lithotripsy Pulse Measurement Errors Due to Nonideal Hydrophone and Amplifier Frequency Responses
}

\author{
Gerald R. Harris, Senior Member, IEEE
}

\begin{abstract}
When using miniature ultrasonic hydrophones to probe the focal region of extracorporeal shock wave lithotripsy devices, the frequency response of the measurement hydrophone and any associated amplifier must be broad enough to minimize pulse distortion. To study the potential effects of bandwidthlimited behavior on lithotripsy pulse measurements, a mathematical model used previously for diagnostic-type pulses was modified. Several parameters of a simulated lithotripsy pulse were compared before and after being filtered by hydrophone and amplifier response functions, both separately and in combination. Errors were computed for the peak positive and negative pressures, rise time, pulse duration, and pulse intensity integral as functions of hydrophone and amplifier bandwidths. Although most of the energy in a shock wave pulse lies at frequencies below a few megahertz, significant errors can occur unless measurement bandwidths are much wider. For example, for a 20-ns rise-time pulse, the bandwidths of the hydrophone or amplifier acting separately should extend beyond $40-50 \mathrm{MHz}$ to keep rise time errors below $10 \%$. However, because the two response functions can compensate one another to some extent, accuracy for the combined response can be maintained at lower bandwidths if the amplifier's cut-off frequency is less than the hydrophone's thickness resonance peak.
\end{abstract}

\section{INTRODUCTION}

A $T$ THE FOCUS of an extracorporeal shock wave lithotripsy (ESL) device's shock wave field, pressure pulses are characterized by an initial compressional (positive) half cycle having a rapidly rising leading edge, followed by a more slowly varying rarefactional (negative) half cycle [1]. After the negative pressure peak, the pressure amplitude gradually decreases, either monotonically or with damped oscillations. Because this slowly varying pressure "tail" can persist for many microseconds, most of the energy in an ESL pulse lies at frequencies below a few megahertz. However, at the highest ESL generator settings, positive pressure rise times typically are less than $50 \mathrm{~ns}$, and may be less than 10 ns. Therefore, a wide bandwidth hydrophone and amplifier are necessary to reproduce faithfully all the features of the ESL pulse.

Coleman and Saunders have made useful experimental comparisons of the responses of different hydrophones to a shock wave pulse [2]. However, their study did not include a quantitative analysis of the errors associated with these

Manuscript received June 19, 1991; revised and accepted October 16, 1991. The author is with the Center for Devices and Radiological Health, Food and Drug Administration, 12721 Twinbrook Parkway, HFZ-132, Rockville, MD 20852

IEEE Log Number 9105553.

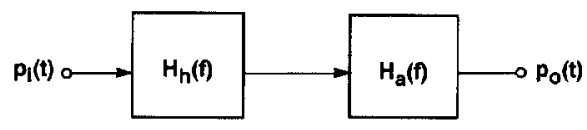

Fig. 1. Block diagram of pulse response model.

measurements. In a previous paper by this author the effects of hydrophone and amplifier frequency response on the measurement of diagnostic ultrasound pulses were studied theoretically [3]. In this paper a similar approach is applied to pulses typical of those encountered in ESL devices. After describing the theoretical model in Section II, various simulation results are presented in Section III. Also considered in Section III are the effects of undersampling by the waveform recording device. Section IV concludes with suggested rules of thumb for choosing hydrophone and amplifier bandwidths.

In this analysis attention is restricted to potential frequency response problems. In practice, however, there are other possible sources of error that should not be overlooked. For example, the effective size and directional response of the hydrophone must be considered, particularly because of the large apertures that can be encountered in ESL devices. Also, nonlinearity of the piezoelectric material, hydrophone cable resonance effects, amplifier impedance, and digitizer resolution all can adversely effect measurement accuracy. Therefore, it should be kept in mind that frequency response, while of fundamental importance, is still only one of several significant factors that can affect the measurement process.

\section{DESCRIPTION OF MODEL}

Fig. 1 contains a block diagram of the pulse response model. The pressure $p_{i}(t)$ represents the ESL pulse as a function of time, $t ; p_{o}(t)$ is the pulse after being filtered by the hydrophone and amplifier response functions, $H_{h}(f)$ and $H_{a}(f)$, respectively, $f$ being frequency. Thus, $p_{o}(t)$ is found from

$$
p_{o}(t)=F^{-1}\left[P_{i}(f) \cdot H_{h}(f) \cdot H_{a}(f)\right]
$$

where $P_{i}(f)$ is the Fourier transform of $p_{i}(t)$, and $F^{-1}$ denotes an inverse Fourier transform. The mathematical forms of $p_{i}(t)$, $H_{h}(f)$, and $H_{a}(f)$ are discussed in the following. 


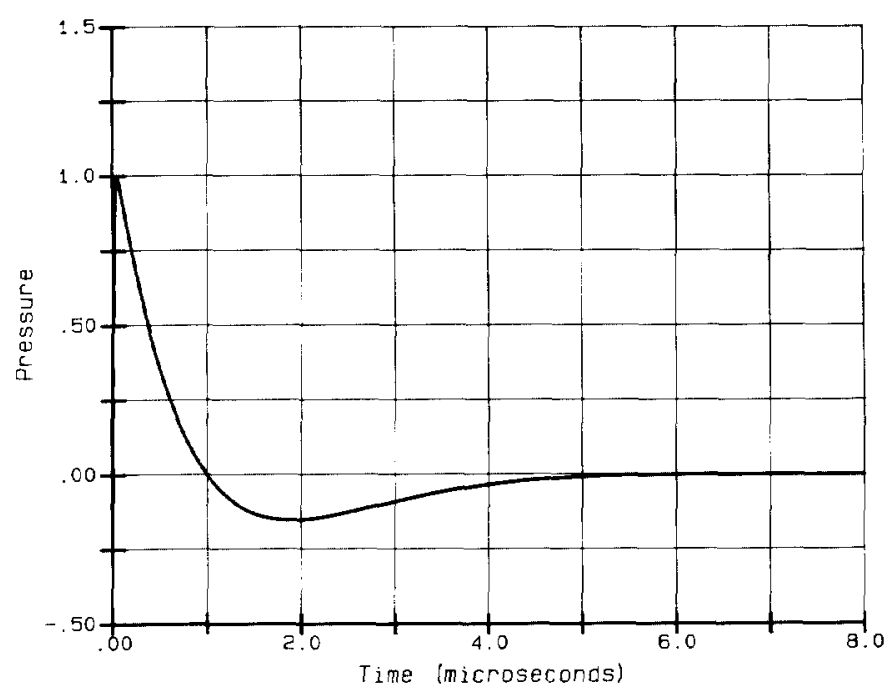

Fig. 2. Simulated ESL pulse $p,(t)$. See Pulse $\# 2$ in Table I.

TABLE I

Values for Constants in (2) Expression for $p ;(t)$, and Calcllated Risf Times, Pulse Widths. and Positive/Negative Pressure Ratios

\begin{tabular}{clllllll}
\hline $\begin{array}{c}\text { Pulse } \\
\#\end{array}$ & $\begin{array}{c}a \\
\left(\mu \mathrm{s}^{-1}\right)\end{array}$ & $\begin{array}{c}j \\
\left(\mu \mathrm{s}^{-1}\right)\end{array}$ & $\begin{array}{c}f \\
(\mathrm{MHz})\end{array}$ & $\begin{array}{c}\boldsymbol{t}_{1} \\
(\mu \mathrm{s})\end{array}$ & $\begin{array}{c}t_{r} \\
(\mathrm{~ns})\end{array}$ & $\begin{array}{c}t_{u} \\
(\mathrm{~ns})\end{array}$ & $p_{c} / p_{2}$ \\
\hline 1 & 1 & 190 & 0.1 & 1.0 & 10 & 350 & 6.9 \\
2 & 1 & 86 & 0.1 & 1.0 & 20 & 370 & 6.6 \\
3 & 1.5 & 96 & 0.5 & 0.6 & 20 & 330 & 4.2 \\
4 & 1.05 & 37.5 & 0.1 & 1.0 & 40 & 390 & 6.5 \\
5 & 3.8 & 27 & 0.7 & 0.4 & 40 & 200 & 8.2 \\
6 & 1.8 & 14 & 0.5 & 0.6 & 80 & 340 & 4.1 \\
\hline
\end{tabular}

\section{A. ESL Pressure Pulse}

The general form for $p_{i}(t)$ used in this study was similar to that described by Church [4]. It comprises the product of falling and rising exponentials and a sinusoid, as in (2):

$$
p_{i}(t)=e^{-\alpha t}\left(1-e^{-\beta t}\right) \sin \left[2 \pi f\left(t_{1}-t\right)\right], \quad t \geq 0 .
$$

Although it is difficult to define a typical pulse shape, proper choices of the constants $\alpha, \beta, f$, and $t_{1}$ in (2) can give pulses that closely mimic those observed experimentally using the best available measurement techniques [1]. These constants were adjusted to achieve desired values for the rise time $\left(t_{r}\right)$, pulse width $\left(t_{w}\right.$, defined as the time between the half amplitude points on the initial positive pressure half cycle), and peak positive to negative pressure ratio $\left(p_{c} / p_{r}\right)$. For example, with $\alpha=1 \mu \mathrm{s}^{-1}, \beta=86 \mu \mathrm{s}^{-1}, f=0.1 \mathrm{MHz}$, and $t_{1}=1 \mu \mathrm{s}$, the pulse in Fig. 2 results. This pulse has a rise time of $20 \mathrm{~ns}$, a pulse width of $370 \mathrm{~ns}$, and a $p_{c} / p_{r}$ ratio of 6.6.

Table I contains data on this pulse and five others used in the simulations. Rise times range from 10 ns to $80 \mathrm{~ns}$. Pulse widths are between $300 \mathrm{~ns}$ and $400 \mathrm{~ns}$, except for Pulse \#5, for which $t_{w}=200 \mathrm{~ns}$. Pressure ratios vary from approximately four to eight.

Values of $p_{i}(t)$ were computed at 4096 equally-spaced points over an $8 \mu \mathrm{s}$ interval, giving an equivalent sampling increment of 1.95 ns. All fast Fourier transform computations were done on 4096-point arrays.
TABLE II

Values of $f_{a}$ (MHz) at Which ERrors Are Less THAN $=5 \%$ AND $=10 \%$ FOR PULSE $\# 2$ IN TABLE I

\begin{tabular}{cccccc}
\hline Error & $p_{r}$ & $p_{v}$ & $p^{2} I$ & $t_{r}$ & $t_{w}$ \\
\hline$< \pm 5 \%$ & 9 & 1 & 5 & 69 & 11 \\
$< \pm 10 \%$ & 5 & $<1$ & 3 & 46 & 7 \\
\hline
\end{tabular}

\section{B. Amplifier and Hydrophone Responses}

The models for the amplifier and hydrophone response functions have been described in [3]. The hydrophone's amplifier response, $H_{a}(f)$, was modeled as a single-pole, low-pass filter with low-pass $(-3 \mathrm{~dB})$ corner frequency, $f_{a}$ (see [3], (4)). The hydrophone response, $H_{h}(f)$, was chosen to approximate the frequency response of polyvinylidene fluoride spot-poled membrane hydrophones. It was derived using Mason's model for a symmetrically loaded piezoelectric receiver [5]. The hydrophone's thickness resonance frequency is denoted by $f_{h}$ (see [3], (2) and (3)).

\section{Pressure Pulse Calculations}

Five quantities were calculated on the pulse before and after filtering; that is, on $p_{i}(t)$ and $p_{o}(t)$. They were peak positive pressure $\left(p_{c}\right)$, peak negative pressure $\left(p_{r}\right)$, pulse pressuresquared integral $\left(p^{2} I\right)$, rise time, and pulse width. Pulse width and rise time were computed via linear interpolation between sample points. An error value, defined as the percent deviation of $p_{o}(t)$ from $p_{i}(t)$, was computed for each of these five quantities.

\section{Simulation Results}

\section{A. Amplifier Response}

First the effect of the amplifier acting alone on $p_{i}(t)$ was considered for 50 values of $f_{a}$ from $2 \mathrm{MHz}$ to $100 \mathrm{MHz}$. The error for the five pulse quantities all approached zero monotonically with increasing frequency. The errors for $p_{r}, p_{c}$, and $p^{2} I$ were negative (i.e., the $p_{o}(t)$ quantities were smaller); those for $t_{r}$ and $t_{w}$ were positive.

As an example, Table II contains frequencies at which the error for the five quantities fall below $\pm 5 \%$ and $\pm 10 \%$ for the pulse in Fig. 2. One can see that, except for rise time, a bandwidth of approximately $10 \mathrm{MHz}$ will result in deviations of $\pm 5 \%$ or less. Also, for a given $f_{a}, p_{r}$, and $p^{2} I$ can be measured more accurately than the other quantities because of their relatively small high-frequency content.

For a pulse $p_{i}(t)$ almost identical to the one in Fig. 2, but with $t_{r}=10 \mathrm{~ns}$ (Table I, Pulse \#1), values of $f_{a}=18$ and $10 \mathrm{MHz}$ result in errors below $\pm 5 \%$ and $\pm 10 \%$, respectively, except for rise time. For the error in $t_{r}$ to be less than or equal to $\pm 5 \%, f_{a}$ must be well above $100 \mathrm{MHz}$; for $\pm 10 \%$, $f_{a}>94 \mathrm{MHz}$.

\section{B. Hydrophone Response}

The variation in error with frequency was more variable for the hydrophone response. The difference is due to the thickness resonance peak in $H_{h}(f)$, a trait common to hydrophones of 


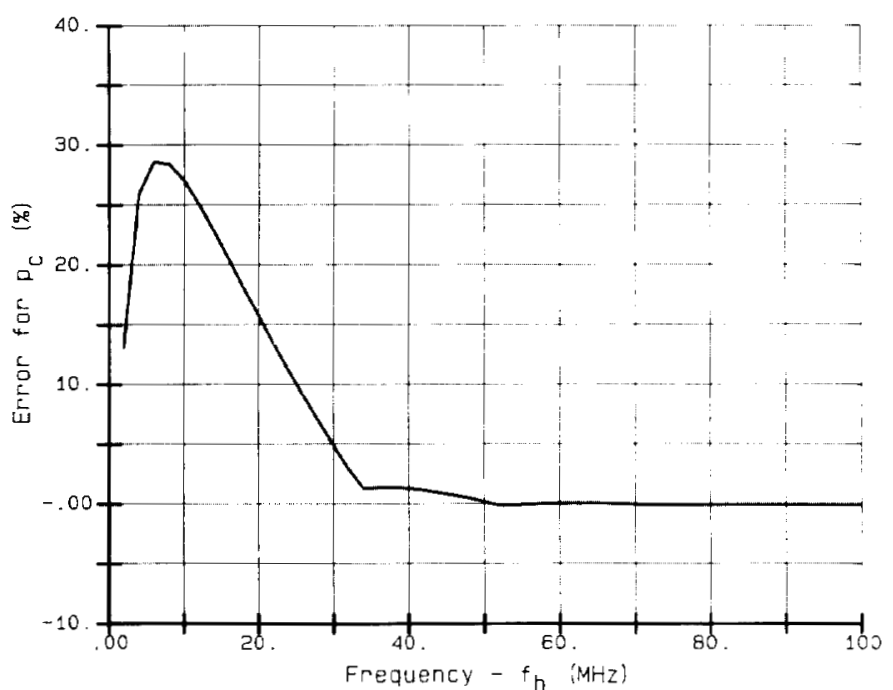

Fig. 3. Error for peak positive pressure $\left(p_{r}\right)$ as a function of hydrophone resonance frequency $\left(f_{h}\right)$ for the pulse in Fig. 2.

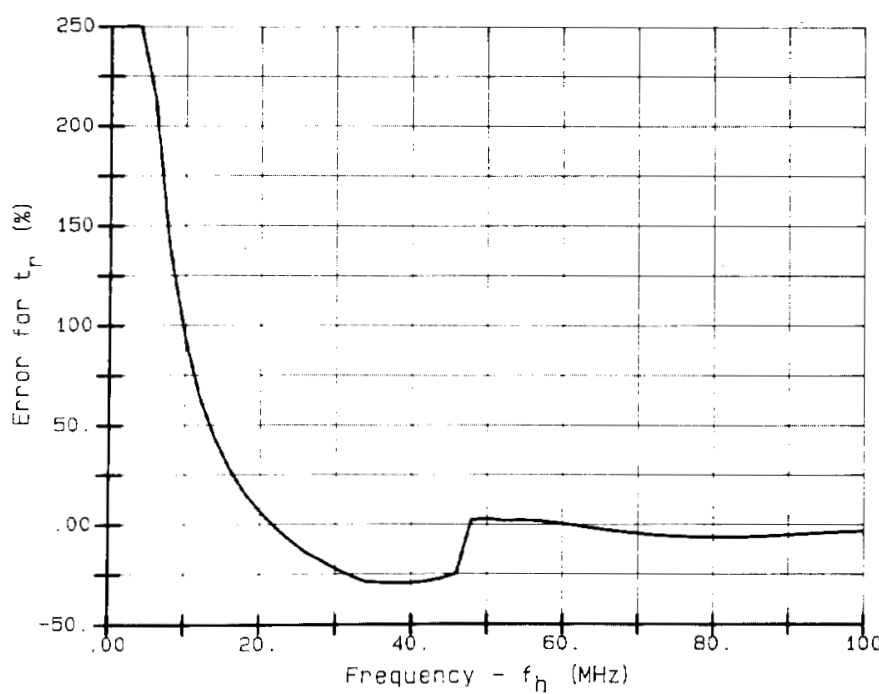

Fig. 4. Error for rise time $\left(t_{i}\right)$ as a function of hydrophone resonance frequency $\left(f_{h}\right)$ for the pulse in Fig. 2. Errors greater than $250 \%$ are not plotted.

the spot-poled membrane type [6], [7]. To illustrate, Figs. 3 and 4 contain plots of the error for $p_{c}$ and $t_{r}$ as a function of $f_{h}$ for the pulse in Fig. 2. In Fig. 3 the error for $p_{c}$ reaches a positive peak at about $5 \mathrm{MHz}$, falls rapidly to less than $2 \%$ at $34 \mathrm{MHz}$, and remains close to zero thereafter. The error for $t_{r}$ in Fig. 4, although initially positive and quite large, passes through $0 \%$ just above $20 \mathrm{MHz}$, reaches a negative peak of about $-30 \%$ at $35-40 \mathrm{MHz}$, then rises quickly to cross the axis again at approximately $50 \mathrm{MHz}$, after which the change is relatively small. There is a negative error peak of about $-5 \%$ at $f_{h}=80 \mathrm{MHz}$.

The changes in both of these plots are due to the oscillations induced in $p_{o}(t)$ by the hydrophone's resonance peak. As an example, Fig. 5 contains the first microsecond of $p_{i}(t)$ and $p_{o}(t)$ for $f_{h}=10 \mathrm{MHz}$. Prominent are the first two oscillatory peaks at approximately $70 \mathrm{~ns}$ and $160 \mathrm{~ns}$ (cf. [2], Fig. 3, which

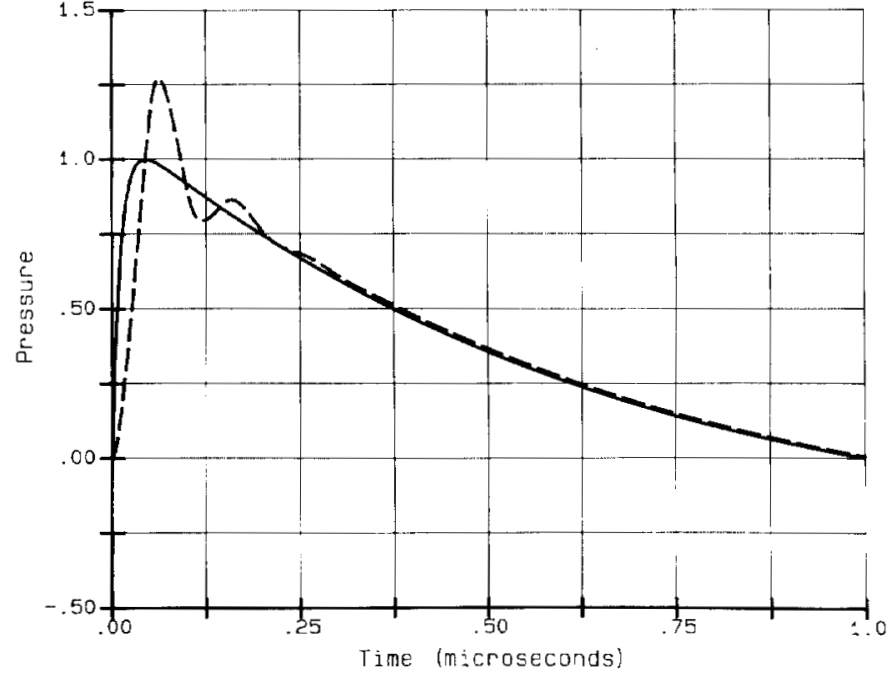

Fig. 5. $p_{i}(t)$ (solid) and $p_{0}(t)$ (dashed) for hydrophone response to Fig. 2 pulse with $f_{h}=10 \mathrm{MHz}$.

TABLE III

VALUES OF $f_{l}(\mathrm{MHz})$ at WhICH ERrors ARE Less THAN $\pm 5 \%$ AND $\pm 10 \%$ FOR PULSE $\# 2$ IN TABLE I

\begin{tabular}{llllll}
\hline Error & $p_{i}$ & $p_{r}$ & $p^{2} I$ & $t_{r}$ & $t_{u}$ \\
\hline$< \pm 5 \%$ & 30 & 1 & 5 & 48 & 31 \\
$< \pm 10 \%$ & 25 & 1 & 2 & 47 & 27
\end{tabular}

displays similar overshoot and ringing from a $50 \mu \mathrm{m}$-thick membrane hydrophone in response to an ESL pulse). As $f_{h}$ is increased the amplitude of the first peak decreases with respect to the second, and the two become equal at $f_{h} \approx 34 \mathrm{MHz}$, the breakpoint in Fig. 3. Between 45 and $50 \mathrm{MHz}$ the first peak falls below $90 \%$ of the second, which accounts for the rapid increase in the rise time of $p_{o}(t)$ just below $50 \mathrm{MHz}$ in Fig. 4. (Recall that rise time is related to the $10 \%$ and $90 \%$ amplitude points on $p_{i}(t)$ or $p_{o}(t)$.)

Table III gives $\pm 5 \%$ and $\pm 10 \%$ error values for $f_{h}$ analogous to Table II. A bandwidth greater than about $50 \mathrm{MHz}$ would keep errors less than $5 \%$ for the pulse in Fig. 2. For the 10 ns rise time pulse, the error plots corresponding to Figs. 3 and 4 have similar shapes, but all the features described previously occur at approximately twice the $f_{h}$ values. Likewise, the $f_{h}$ values in Table III approximately double for $p_{c}, t_{r}$, and $t_{w}$.

\section{Combined Response}

To study the effects of the combined amplifier and hydrophone response, $f_{a}$ was varied from $2 \mathrm{MHz}$ to $100 \mathrm{MHz}$ in steps of $2 \mathrm{MHz}$, for $f_{h}$ values of 20,40 , and $80 \mathrm{MHz}$. These three $f_{h}$ values correspond to membrane hydrophone film thicknesses of approximately 50,25 , and $12 \mu \mathrm{m}$, respectively.

Plots of the error versus $f_{a}$ in response to the Fig. 2 pulse are given in Fig. 6(a)-(d) for $p_{c}, p^{2} I, t_{r}$, and $t_{w}$. Unlike the equivalent plots for $H_{k}(f)$ in Figs. 3 and 4 , here all traces are single-valued. Also, the final values (i.e., the $f_{a}=100 \mathrm{MHz}$ values) are not necessarily the lowest values, because most of the curves cross $0 \%$ at a value of $f_{a}$ less than or approximately 


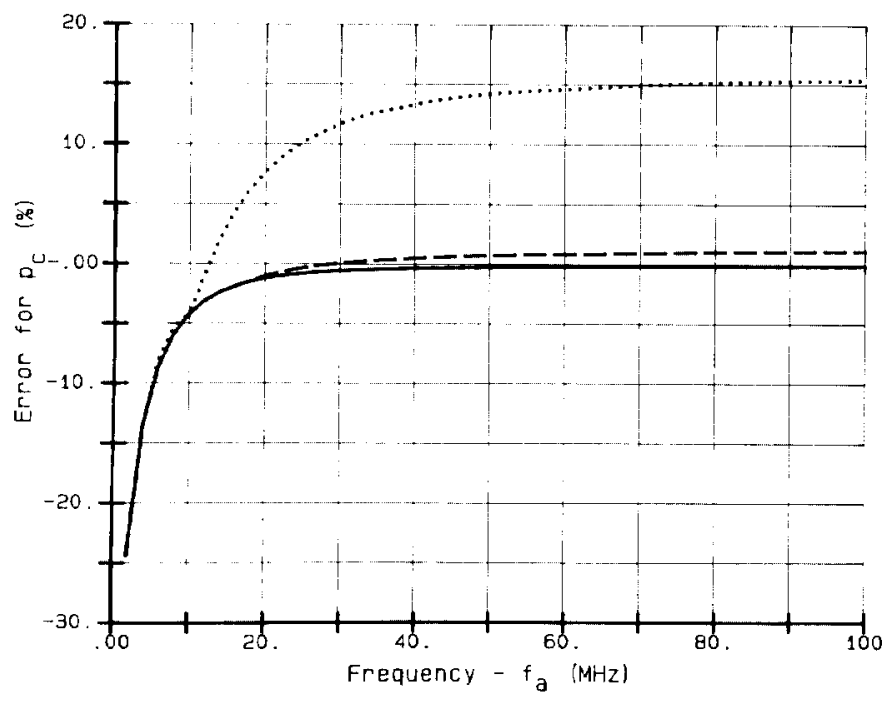

(a)

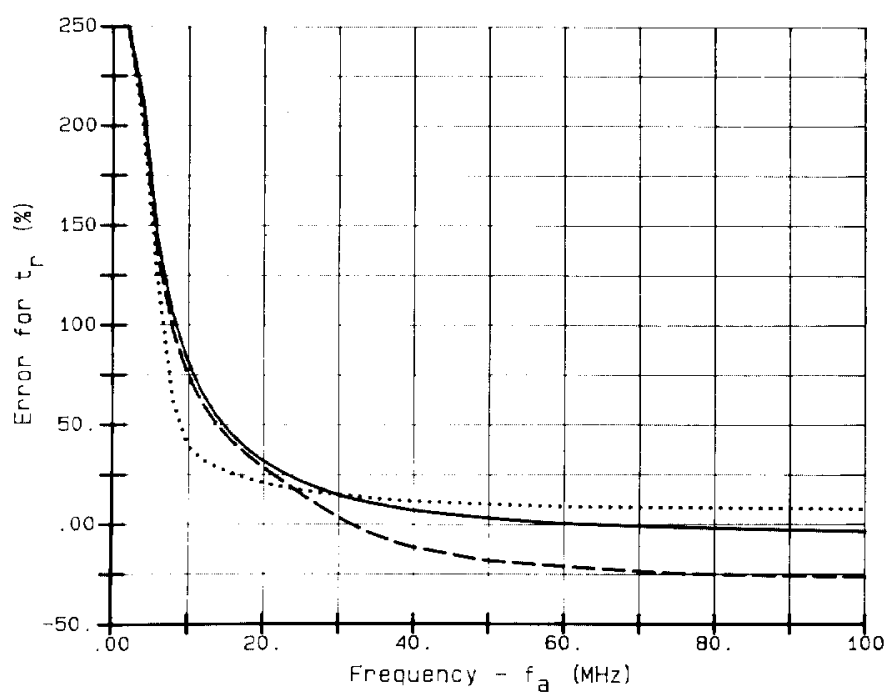

(c)

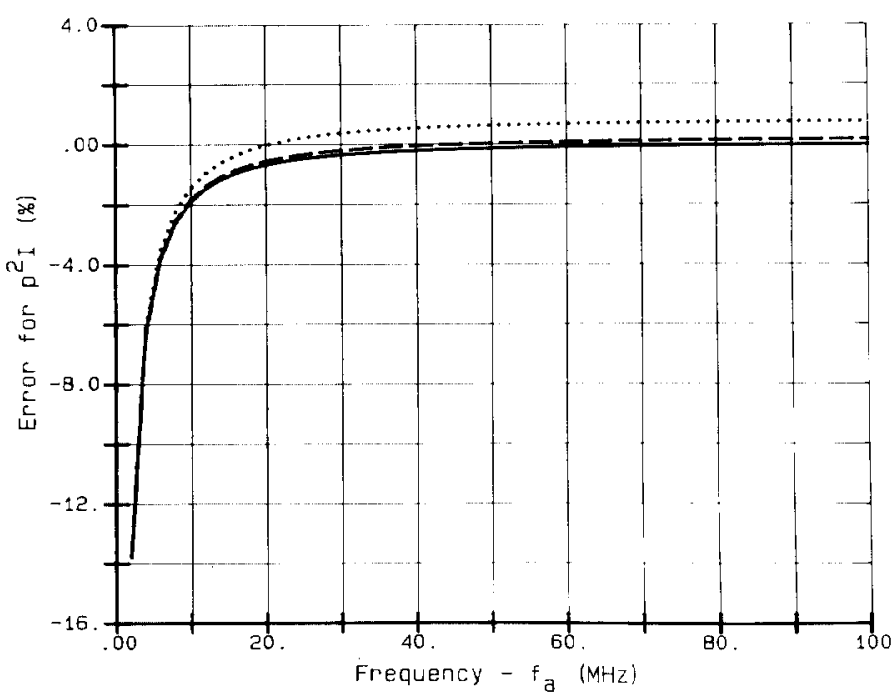

(b)

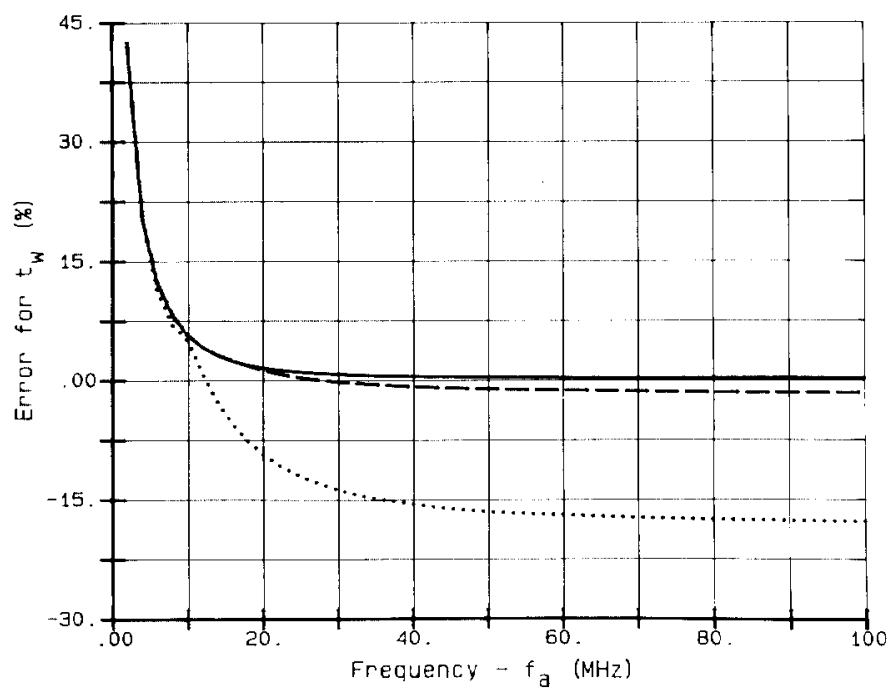

(d)

Fig. 6. Error versus $f_{a}$ for combined hydrophone/amplifier response to Fig. 2 pulse at $f_{h}=20 \mathrm{MHz}$ (dotted), $f_{h}=40 \mathrm{MHz}$ (dashed), and $f_{h}=80 \mathrm{MHz}$ (solid). (a) $p_{c}$; (b) $p^{2} I$; (c) $t_{r}$; (d) $t_{u}$. In (c), errors greater than $250 \%$ are not plotted.

equal to $f_{h}$. Furthermore, for the case of rise time in Fig. 6(c), the $f_{a}=100 \mathrm{MHz}$ value for $f_{h}=20 \mathrm{MHz}$ is less than the value for $f_{h}=40 \mathrm{MHz}$; that is, the hydrophone with the smaller bandwidth seems to be more accurate. Though not an intuitive result, it is consistent with Fig. 4, which can be considered an error plot for $t_{r}$ versus $f_{h}$ at $f_{a}=\infty$. (Compare the errors at $20 \mathrm{MHz}$ and $40 \mathrm{MHz}$ in Fig. 4.)

Fig. 7 contains the first microsecond of $p_{i}(t)$ and $p_{o}(t)$ for $f_{a}=30 \mathrm{MHz}$ and $f_{h}=40 \mathrm{MHz}$, where $p_{i}(t)$ is the pulse in Fig. 2. In this case the error for $t_{r}$ is approximately $3 \%$, and the other errors are all within $\pm 1 \%$. For the 10 ns rise time pulse, $f_{a}=60 \mathrm{MHz}$ and $f_{h}=80 \mathrm{MHz}$ give similar results.

The error for $p_{r}$ was not plotted in Fig. 6 because, for values of $f_{a}$ greater than 1-2 $\mathrm{MHz}$, the error was less than $5 \%$. The only situation studied in which the error for $p_{r}$ exceeded $5 \%$ at $f_{a}=2 \mathrm{MHz}$ was when $f$ in (2) was increased, as could be encountered with piezoelectric lithotripters [1]. For example, using Pulse \#3 in Table I, which has a frequency $f$ of $0.5 \mathrm{MHz}$
(Fig. 8), the error for $p_{r}$ was between $5 \%$ and $10 \%$ at $f_{a}=2$ MHz. In no case considered in Table I was the error for $p_{r}$ significantly greater than $10 \%$ until $f_{a}$ fell below $2 \mathrm{MHz}$.

\section{Errors Induced by Undersampling}

With a sampling increment of 1.95 ns (Section II-A), the sampling rate for the various ESL pulses modeled was approximately $500 \mathrm{MHz}$. To examine what effect decreasing this rate could have on pulse measurements, rise time and peak positive pressure values for sampling rates at 50,100 , and $250 \mathrm{MHz}$ were compared to the "true" values at $500 \mathrm{MHz}$. Maximum rise time errors for 10 ns and 20 ns rise time pulses (Pulses \#1 and \#2 in Table I) are given in Table IV.

As can be seen from Table IV, rise time measurements can be affected significantly by undersampling the time waveform. As a rule of thumb, the sampling interval should be at least five times smaller than the measured rise time. 


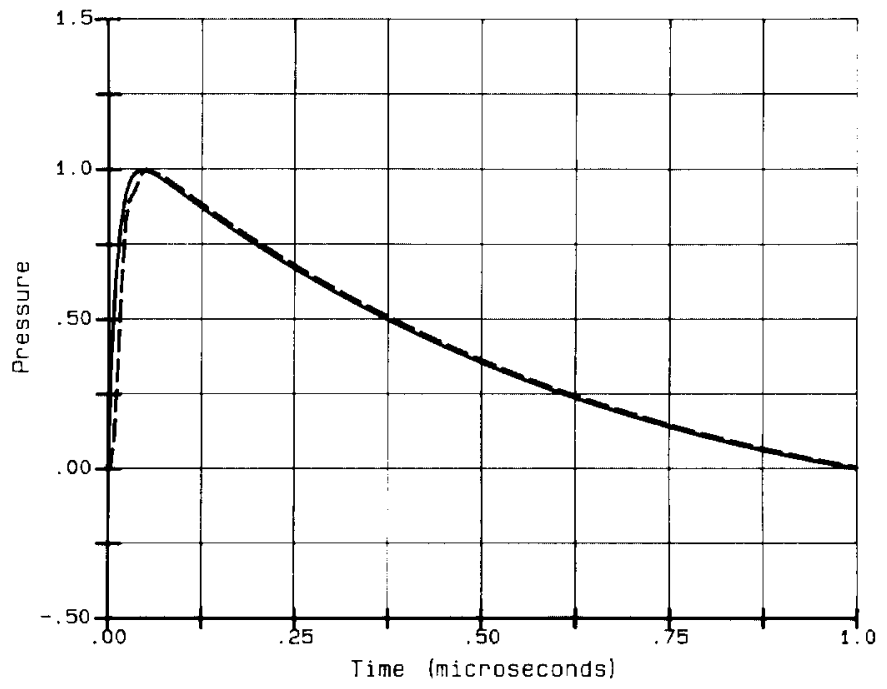

Fig. 7. $p_{i}(t)$ (solid) and $p_{0}(t)$ (dashed) for combined hydrophone/amplifier response to Fig. 2 pulse with $f_{a}=30 \mathrm{MHz}$ and $f_{h}=40 \mathrm{MHz}$.

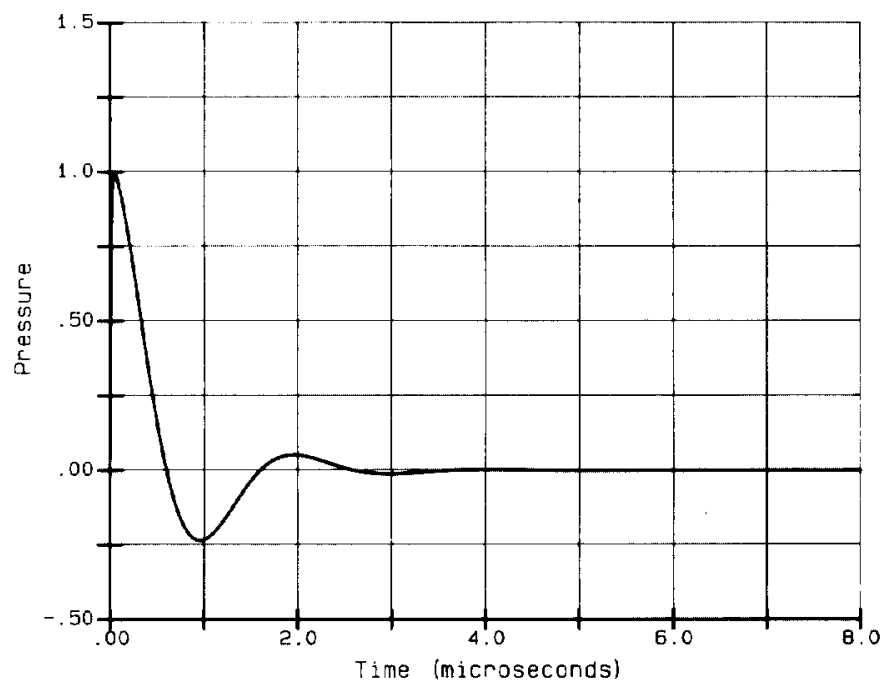

Fig. 8. Simulated ESL pulse $p_{i}(t)$. See Pulse \#3 in Table I.

TABLE IV

MAXimum Rise Time ERrors Due to Undersampling FOR PULSES WITH $t_{r}=10$ ns AND 20 ns

\begin{tabular}{ccc}
\hline Sampling Rate & \multicolumn{2}{c}{ Error } \\
\cline { 2 - 3 }$(\mathrm{MHz})$ & $t_{r}=10 \mathrm{~ns}$ & $t_{r}=20 \mathrm{~ns}$ \\
\hline 50 & $210 \%$ & $62 \%$ \\
100 & $62 \%$ & $22 \%$ \\
250 & $15 \%$ & $4.0 \%$ \\
\hline
\end{tabular}

Corresponding errors for $p_{c}$ were quite small, exceeding $1 \%$ only at $50 \mathrm{MHz}$ for the $10 \mathrm{~ns}$ rise time pulse. This small error is due to the fall time for the initial positive pressure half cycle being much larger than the rise time, typically being hundreds of nanoseconds in duration.

\section{CONCLUSION}

The usefulness of the analysis in this paper is dependent on how well the functional forms of $p_{i}(t), H_{a}(f)$, and $H_{h}(f)$ match real world situations. With regard to $p_{i}(t)$, Figs. 2 and 8 , as well as the other pulses represented in Table I, are similar to experimentally observed pulses. In practice ESL pulses having rise times less than the $10 \mathrm{~ns}$ lower limit considered here are conceivable. For example, it has been shown that shock wave rise times can be on the order of 1 ns or less [8]. However, accurate measurements below about $10 \mathrm{~ns}$ are beyond the capabilities of current hydrophones [6]. Furthermore, it is not clear how much such refined knowledge would add to safety or effectiveness studies of ESL devices. Still, it should be kept in mind that if a measurement with the best of conventional hydrophones yields a rise time of $10 \mathrm{~ns}$, the actual rise time could be much less.

With regard to the hydrophone and amplifier response models, the $H_{h}(f)$ used is applicable primarily to membrane type piezopolymer hydrophones. However, needle type hydrophones displaying no resonance peak have been used to measure ESL pulses [9], [10]. The response of one of these needle probes begins rolling off at about $10 \mathrm{MHz}$ [9]. If the frequency response of this hydrophone is approximated by the amplifier response $H_{a}(f)$ with $f_{a}=10 \mathrm{MHz}$, then the results in Section III-A can be used to estimate its performance. (Note that in [2], at least one of these needle probes has been found to have diminished low frequency response, a feature not examined here.)

Based on simulations of the pulses in Table $I$, the two rules of thumb that follow can be used to assist in choosing hydrophone and amplifier bandwidths, given an expected value for the rise time.

1) Bandwidths equal to or greater than $1 / t_{r}$ will keep rise time errors less than about $10 \%$, and other pulse quantity errors less than about $2 \%$.

2) Bandwidths equal to or greater than $1 / 2 t_{r}$ will keep rise time errors less than about $30 \%$, and other pulse quantity errors less than about $15 \%$.

In Section III-C an example was given of how accuracy for the combined hydrophone/amplifier response could be maintained at lower bandwidths by choosing an $f_{a}$ a little less than $f_{h}$ (In the example, $f_{a}=0.75 f_{h}$ ). However, optimal selection of $f_{a}$ and $f_{h}$ requires some a priori knowledge of the pulse to be measured.

In addition to these bandwidth considerations, the sampling rate of any waveform digitizer used should be greater than approximately $5 / t_{r}$ to keep rise time errors due to undersampling small (less than a few percent).

\section{REFERENCES}

[1] A. J. Coleman and J. E. Saunders, "A survey of the acoustic output of commercial extracorporeal shock wave lithotripters," Ultrasound in Med. \& Biol., vol. 15, no. 3, pp. 213-227, 1989.

[2] - "A comparison of PVdF hydrophone measurements in the acoustic field of a shock wave source," in Extra- und Intrakorporale Lithotripsie bei Harn-, Gallen-, Pankreas- und Speichelsteinen, C. Ell, M. Marberger, and P. Berlien, Eds. New York: Georg Thieme Verlag, 1990, pp. 14-22 (in English).

[3] G. R. Harris, "A model of the effects of hydrophone and amplifier frequency response on ultrasound exposure measurements," IEEE Trans. Ultrason., Ferroelec., Freq. Contr., vol. 38, no. 5, pp. 413-417, 1991.

[4] C. C. Church, "A theoretical study of cavitation generated by an extracorporeal shock wave lithotripter," J. Acoust. Soc. Am., vol. 86, 
no. 1, pp. 215-227, 1989.

[5] W. P. Mason, Electromechanical Transducers and Wave Filters, second ed. Princeton, NJ: Van Nostrand, 1948, p. 200ff, p. $300 \mathrm{ff}$.

[6] D. R. Bacon, "Characteristics of a pvdf membrane hydrophone for use in the range 1-100 MHz," IEEE Trans. Sonics Ultrason., vol. SU-29, no. 1 , pp. $18-25,1982$

[7] G. R. Harris, "Hydrophone measurements in diagnostic ultrasound fields," IEEE Trans. Ultrason., Ferroelec., Freq. Contr., vol. 35, no. 2, pp. 87-101, 1988.

[8] D. T. Blackstock, "Thermoviscous attenuation of plane, periodic, finiteamplitude sound waves," J. Acoust. Soc. Am., vol. 36, no. 3, pp. $534-542,1964$.

[9] M. Platte, "A polyvinylidene fluoride needle hydrophone for ultrasonic applications," Ultrason., vol. 23, no. 3, pp. 113-118, 1985.

[10] P. A. Lewin and A. S. DeReggi, "Short range applications," in The Applications of Ferroelectric Polymers, T. T. Wang, J. M. Herbert, and A. M. Glass, Eds. New York: Chapman and Hall, 1988, p. 186.

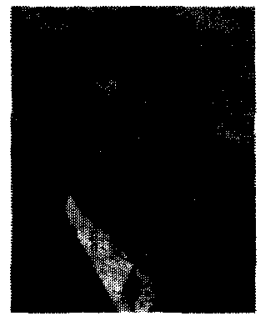

Gerald R. Harris (M'72-S'76-M'79-SM'82) was born in Jacksonville, NC, on November 22, 1945. He received the B.E.E. degree in 1967 from the Georgia Institute of Technology, Atlanta, the M.S. degree in biological engineering in 1971 from the Rose-Hulman Institute of Technology, Terre Haute, $\mathrm{IN}$, and the Ph.D. degree in electrical engineering in 1982 from the Catholic University of America, Washington, $D C$

Since 1967, he has been employed by the U.S. Public Health Service, and he is currently with the Food and Drug Administration's Center for Devices and Radiological Health, where his main activities comprise the theoretical and experimental evaluation of medical ultrasound transducers and systems.

Dr. Harris is a member of the Acoustical Society of America, the American Institute of Ultrasound in Medicine, and Sigma Xi. 